\title{
Antibiotics in surgical wards: use or misuse? A newly industrialized country's perspective
} \author{
Adeeba Kamarulzaman ${ }^{2}$ \\ ${ }^{1}$ Department of Primary Care Medicine, University of Malaya, Kuala Lumpur, Malaysia \\ ${ }^{2}$ Department of Medicine, University of Malaya, Kuala Lumpur, Malaysia \\ ${ }^{3}$ Department of Surgery, University of Malaya, Kuala Lumpur, Malaysia \\ ${ }^{4}$ Department of Medical Microbiology, University of Malaya, Kuala Lumpur, Malaysia \\ ${ }^{5}$ Department of Microbiology and Infectious Diseases, Royal Perth Hospital, Perth, Australia \\ ${ }^{6}$ Centre for Medicine Use and Safety, Monash University, Victoria, Australia
}

Mun Kit Lim ${ }^{1}$, Pauline Siew Mei Lai ${ }^{1}$, Sasheela Sri La Sri Ponnampalavanar ${ }^{2}$, Sharifah Faridah Syed Omar $^{2}$, Nur Aishah Taib ${ }^{3}$, Mohamad Yasim Yusof ${ }^{4}$, Claire Marie Italiano ${ }^{5}$, David Chee Ming Kong ${ }^{6}$,

\begin{abstract}
Introduction: Studies exploring the appropriateness of therapeutic antibiotic use among surgical patients are limited, particularly in developing countries. Therefore, the aim of our study was to determine the appropriateness of antibiotics prescribed in a surgical setting in Malaysia.

Methodology: A prospective observational study was conducted in two surgical wards at a tertiary hospital in Malaysia from November 2012-July 2013. Data was collected using a case report form. The appropriateness of antibiotic therapy was based on compliance with either the Malaysian National Antibiotic Guidelines 2008 or International Clinical Practice Guidelines and determined by an expert panel (consisting of two infectious disease consultants and a pharmacist).

Results: Over the study period, a total of 593 antibiotic courses were prescribed for 129 patients (4.6 \pm 3.4 antibiotics/patient). Only 34 (26.4\%) patients received appropriate antibiotic therapy, whilst 95 (73.6\%) patients received at least one course of inappropriate antibiotic therapy. The prevalence of inappropriate antibiotic use was $214(66.3 \%)$ and $55(42.0 \%)$ for prophylactic and therapeutic purposes, respectively. The most common causes of inappropriate prophylactic antibiotics were inappropriate timing 20 (36.4\%) and inappropriate duration of prophylaxis 19 (34.5\%). In cases of inappropriate timing, $9(45 \%)$ were administered too late while $6(30 \%)$ were too early. . In contrast, inappropriate choice of antibiotics $(42.1 \%)$ and inappropriate indication $(40.7 \%)$ were the most common reasons encountered for inappropriate therapeutic antibiotics.

Conclusion: Our study suggests considerable inappropriate use of both prophylactic and therapeutic antibiotics in the surgical wards; highlighting an urgent need for antibiotic stewardship initiatives in this setting.
\end{abstract}

Key words: antibiotic stewardship; surgical; appropriateness; antibiotics; Malaysia.

J Infect Dev Ctries 2015; 9(11):1264-1271. doi:10.3855/jidc.6731

(Received 12 February 2015 - Accepted 30 April 2015)

Copyright $(2015$ Lim et al. This is an open-access article distributed under the Creative Commons Attribution License, which permits unrestricted use, distribution, and reproduction in any medium, provided the original work is properly cited.

\section{Introduction}

The term "newly industrialized country" is a socioeconomic classification applied to countries whose economies have not yet reached developed country status but have outpaced their developing counterparts [1]. Since 2011, Malaysia has been categorized as a newly industrialized country. However, there remains a paucity of information regarding antibiotic prescribing when compared to developed countries, particularly in the surgical setting. Antibiotic stewardship programs (ASP) designed to combat the development of resistance are almost non-existent.
Antibiotics are frequently used in surgical patients as approximately $30 \%$ of patients undergoing surgery will develop post-operative surgical site infections (SSI) [2]. However, prophylactic antibiotics should be administered in a timely manner, and therapeutic antibiotics must be used judiciously $[3,4]$. Prevention and management of infection is imperative as postoperative SSI alone can prolong hospitalization, double readmission rates, and triple the cost of treatment [5]. Inappropriate use of antibiotics, however, can increase resistance towards antibiotics, morbidity, mortality and cost of health-care $[6,7,8,9]$. This study determined the appropriateness of antibiotic prescription among surgical patients and will act as an 
important guide in the development and implementation of an effective ASP.

\section{Methodology}

A prospective observational study was conducted in two surgical wards at a tertiary hospital in Malaysia. Surgical patients prescribed antibiotics from November 2012-July 2013 were included. Each patient was followed up until discharge or in-hospital death. For patients readmitted during the study period, only the first admission was included to avoid potential confounders related to readmission. Immunocompromised patients were excluded.

On alternate days, the researcher reviewed patient medical records by alternating between the left and right wings of the ward. If the patient satisfied the inclusion criteria, informed consent was obtained. Patient demographic information, pattern of antibiotic use, microbiological and clinical outcomes were collected. Information regarding readmission within 30-days was obtained via medical records or via patient telephone call if this information was not available.

The primary outcome measure was appropriateness of antibiotics prescribed (Table 1). The sample size required for this study was 385 antibiotic courses (confidence level $=95 \%$ ) based on a rate of inappropriate antibiotic use of $50 \%$ [10]. Secondary outcome measures included pattern of antibiotic use, antibiotic resistance pattern, and clinical outcomes. Appropriateness of antibiotics was determined based on compliance with guidelines and evaluated by an expert panel (consisting of two infectious disease consultants and a pharmacist) (Figure 1) $[4,11,12]$. The amount of antibiotics used was measured in defined daily doses per 1000 occupied bed-days (DDD/1000 BD) [13]. Antibiotic resistance data was obtained from the hospital's laboratory information system. Specimens assessed included cultures from blood, intra-operative specimens, sputum, urine and any other culture from a sterile site in the setting of suspected clinical infection.

Table 1. Definitions used to determine the appropriateness of antibiotics prescribed

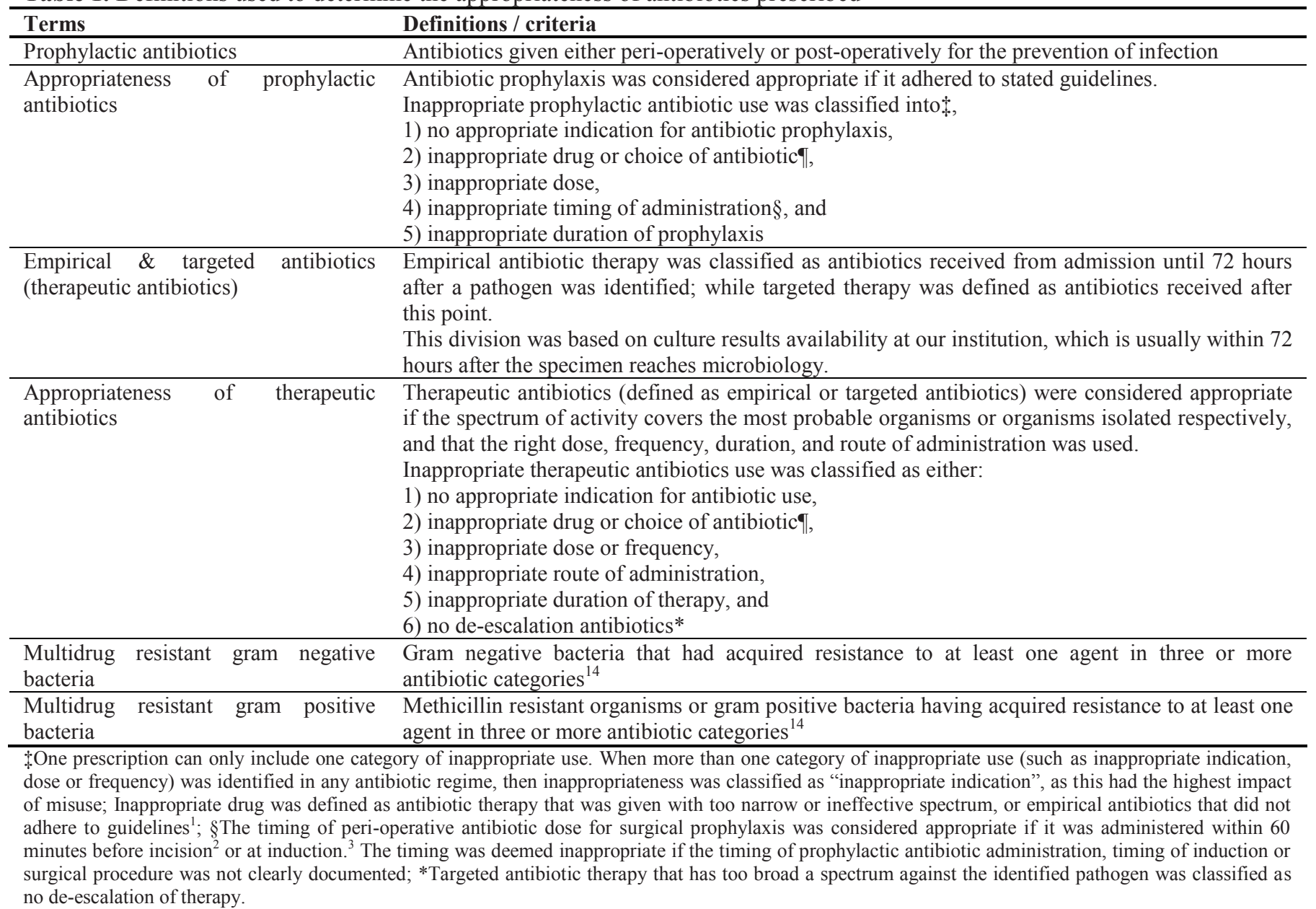


Figure 1. Flow chart on the evaluation of the appropriateness of antibiotic use in surgery

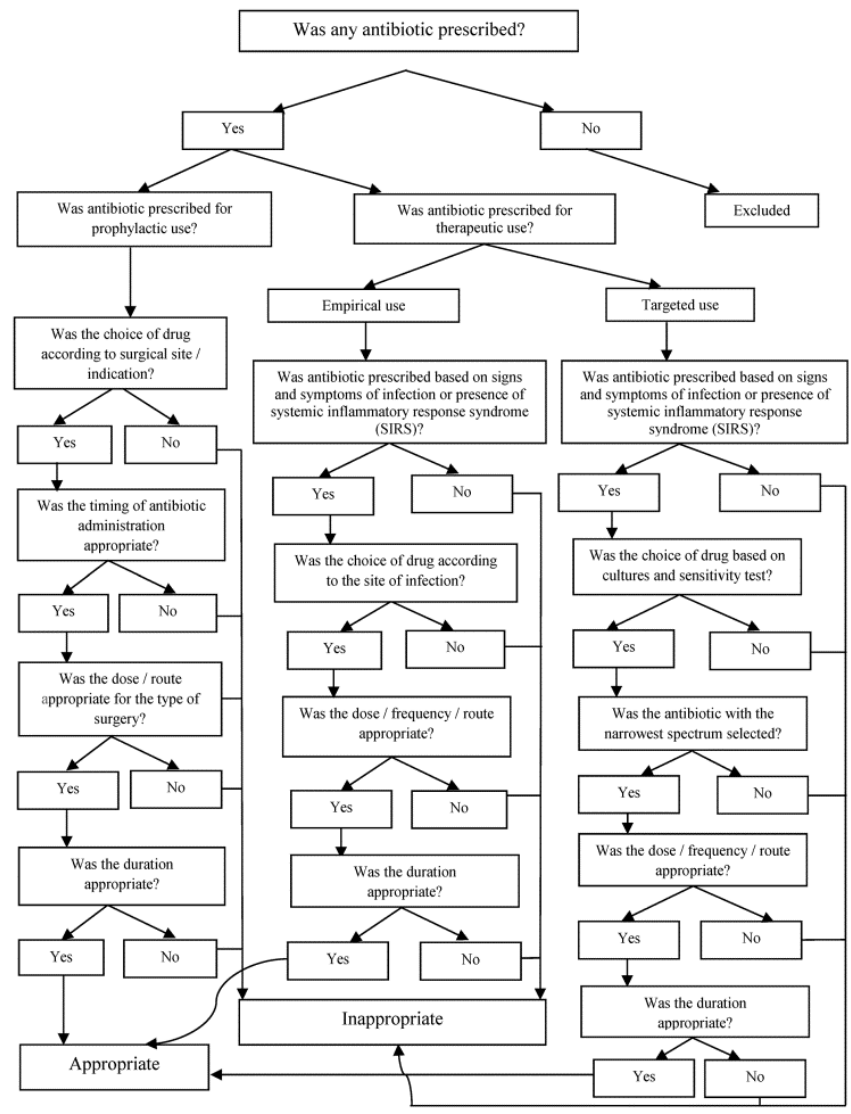

Evaluation of the appropriateness of antibiotic use in surgery was based on the Malaysian National Antibiotic Guidelines 2008 and International Clinical Practice Guideline ${ }^{4,11,12}$ which was reviewed by an expert panel (which consisted of two infectious disease consultants and a pharmacist). Surgeons were invited to provide input when antibiotics prescribed were classified as inappropriate.

Isolates of the same organism having identical antibiotic sensitivity patterns obtained from the same sampling site within seven days were excluded. The report of normal flora from a culture was assumed to be a negative culture. The proportion of cultures with multidrug resistant (MDR) bacteria was analyzed to establish the pattern of bacterial resistance [14].

\section{Data Analysis and Ethics}

All data were entered into IBM SPSS version 20 (IBM Corporation, Armonk, NY, USA). Continuous data were analyzed using Mann Whitney-U test. Categorical data were assessed using Fisher's exact test. Ethics approval was obtained from the University Malaya Ethics Committee (approval no. 932.2).

\section{Results}

\section{Demographics}

Over the study period, a total of 593 antibiotic courses were prescribed for 129 patients. Eighty-four patients $(65.1 \%)$ had surgical interventions during their stay in the surgical ward, with $42(32.6 \%)$ given peri-operative antibiotic prophylaxis. The remaining $45(34.9 \%)$ patients were admitted for dressings, suture removal, wound breakdown or other nonoperative management (Table 2). Only 34 (26.4\%) patients received appropriate antibiotic therapy, whilst $95(73.6 \%)$ patients received at least one course of inappropriate antibiotic therapy (Figure 2).

\section{Antibiotic use for surgical prophylaxis}

Out of 83 courses of prophylactic antibiotic therapy, 67 courses were prescribed peri-operatively while 16 courses were prescribed post-operatively. Inappropriate antibiotic use for prophylaxis occurred in 55 cases $(66.3 \%)$. The most common reasons for inappropriate prophylactic antibiotics use were inappropriate timing 20/55 (36.4\%), inappropriate duration 19/55 (34.5\%) and inappropriate choice of antibiotic 9/55 (16.4\%) In cases of inappropriate timing, 9/20 (45\%) were due to late administration (after incision or intubation), 6/20 (30\%) due to too early administration and 5/20 (25\%) due to no proper documentation on the time of surgery or antibiotic administration.

Figure 2. Overview regarding the appropriateness of antibiotic therapy in surgical wards

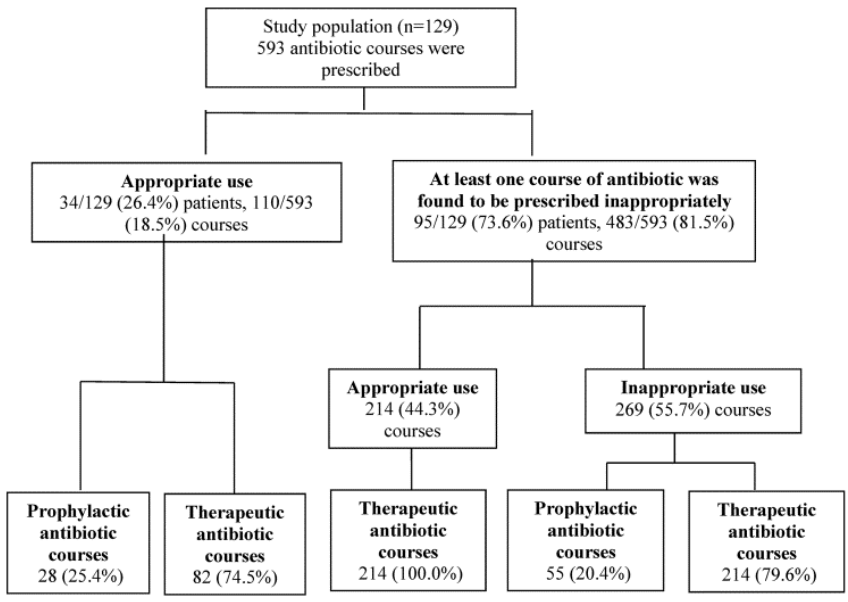

Overall, therapeutic antibiotics were more frequently prescribed appropriately than prophylactic antibiotics [therapeutic antibiotics $296(91.4 \%)$ versus prophylactic antibiotics $28(8.6 \%), \mathrm{p}<0.001]$ 
Table 2. Demographic characteristics of patients $(n=129)$

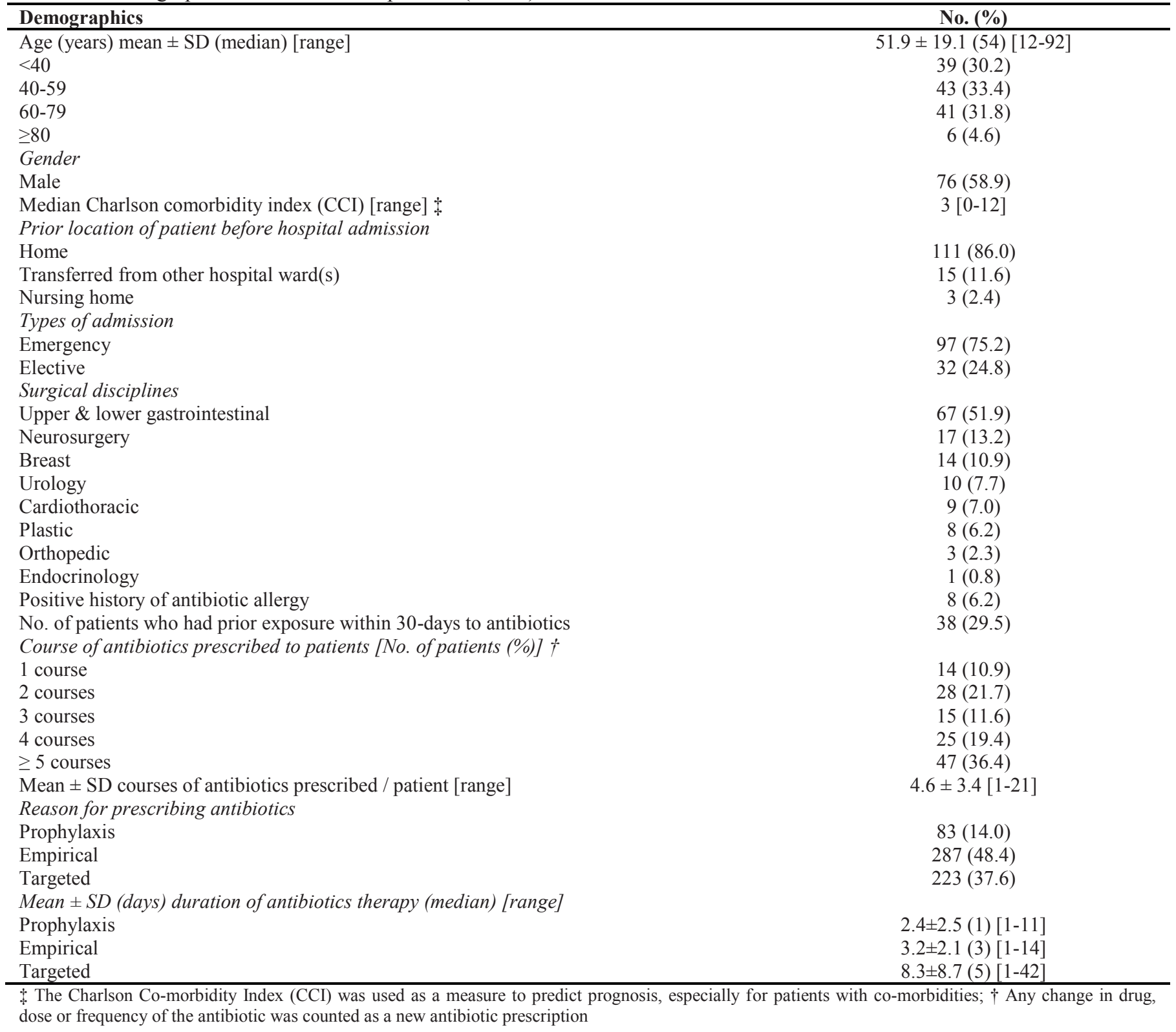

Table 3. Clinical outcomes of patients

\begin{tabular}{lc}
\hline & No. (\%) \\
\hline Surgical site infection rate per patient & $11 / 84(13.1)$ \\
Median (days) duration of hospitalization [range] & $10[1-158]$ \\
Readmission within 30 days to the surgical wards & $25(19.4)$ \\
Readmission due to infection & $8(6.2)$ \\
Death within 30 days & $11(8.5)$ \\
30 days all-cause mortality per 1000 patients* & 85.3 \\
\hline
\end{tabular}

\footnotetext{
*All-cause mortality formula $=$ no. of death/total population $\mathrm{x} 1000$ patients.
} 
Antibiotics use in empirical and targeted therapy

Overall, 214/510 (42.0\%) therapeutic antibiotics were prescribed inappropriately, with 122/214 (57.0\%) and 92/214 (43.0\%) prescribed for empirical and targeted use, respectively. Patients requiring urological procedures had the highest prevalence of inappropriate use $(68.8 \%)$, followed by cardiothoracic surgery $(62.1 \%)$ and plastic surgery $(45.0 \%)$ cases. The most common reasons for inappropriate therapeutic antibiotic were inappropriate drug 90 (42.1\%), no appropriate indication $87(40.7 \%)$ and inappropriate duration of treatment $19(8.9 \%)$. Aminoglycosides 5 (66.7\%), second generation cephalosporins $42(60 \%)$ and carbapenems $23(56.1 \%)$ were most commonly inappropriately prescribed.

\section{Pattern of antibiotic use in surgery}

Overall, the most commonly prescribed classes of antibiotics were third generation cephalosporins (198.1 DDD/1000 patient days), imidazole-derivatives (185.2 $\mathrm{DDD} / 1000$ patient days) and penicillin combinations, including betalactamase-inhibitors (185 DDD/1000 patient days). The most frequently prescribed individual agents were metronidazole, vancomycin, and ceftriaxone for 185.2, 133.2 and 109.7 DDD/1000 patient days respectively.

\section{Antibiotic resistance pattern}

More gram negative organisms were identified in isolates as compared to gram positive organisms. Gram negative organisms were predominantly isolated from the gastrointestinal and hepatobiliary system in 7 $(63.6 \%)$ cultures, wound in $64(47.8 \%)$ cultures, respiratory system in $23(36.5 \%)$ cultures, and catheter in $9(20.5 \%)$ cultures. Extended spectrum betalactamase (ESBL) producing Enterobactericeae 33 (25.2\%), MDR Acinetobacter baumanii 16 (12.2\%) and MDR Pseudomonas aeruginosa 3 (2.3\%) were the most frequent resistant gram negative pathogens.

Gram positive organisms were most commonly isolated from blood and cerebrospinal fluid in 41 $(18.7 \%)$ and $2(7.4 \%)$ respectively. The most frequently resistant gram positive organisms were methicillin-resistant Staphylococcus aureus 34 (26\%), methicillin-resistant coagulase-negative staphylococci $26(19.8 \%)$ and ampicillin-resistant enterococci 12 $(9.2 \%)$.

\section{Clinical outcomes of patients}

Of the 11 patients who died, all were prescribed at least one inappropriate antibiotic course and 5 (45.5\%) had sepsis reported as a contributing factor in the cause of death. The median duration of hospitalization was 10 days with all-cause 30-day mortality of 85.3 per 1000 patients with inappropriate antibiotic use not significantly impacting on these outcomes (Table 3).

\section{Discussion}

The rates of inappropriate use of antibiotics in this setting in Malaysia reflect those found previously in Asian countries: $66.3 \%$ and $42.0 \%$, for prophylactic and therapeutic antibiotics, respectively. Studies conducted in Asian countries have generally reported higher prevalence of inappropriate antibiotic use for prophylaxis (48-100\%) when compared to developed countries $\quad(23 \cdot 4 \%-55 \cdot 2 \%) \quad[15,16,17,18,19]$. Inappropriate timing of antibiotic administration (36.4\%) and inappropriate duration (34.5\%) were found to be the main reasons for inappropriate prophylactic antibiotic which was also similar to previous studies $[17,18,20]$. The inappropriate timing of administration in our setting was most likely due to the lack of local institutional guidelines. Single dose perioperative antibiotics, and when necessary, postoperative prophylaxis for no more than 24 hours are recommended [12]. The $16(19.3 \%)$ courses of antibiotics given post-operatively may reflect a practice that could be easily targeted for intervention. Unnecessarily prolonging the duration of prophylaxis does not necessarily lead to better outcomes, but instead predisposes to selective pressure for resistant bacteria [18].

Additionally, it was also noted that the timing of prophylactic antibiotic administration in our setting was poorly documented. One reason for this may be that documentation of prophylactic antibiotic administration is performed by anaesthesiologists on induction forms, whereas the time of surgical incision is documented elsewhere by surgeons. Standardized forms to record the time of administration of prophylactic antibiotics and the time of surgical incision could be developed to overcome this problem in conjunction with prescriber education.

The inappropriate prescription of therapeutic antibiotics may be due to the lack of institutional antibiotic guidelines, as infectious disease ward rounds and formulary restrictions of antibiotic were already in place. Due to the heterogeneity of inappropriate empirical and targeted antibiotic use between different surgical disciplines, guidelines should take into account the requirements of each discipline.

The use of third generation cephalosporins, imidazole derivatives and second generation cephalosporins resemble the patterns seen in other 
Asian countries where broad spectrum cephalosporin use is predominant [16,19,21,22]. Contrary to this, many developed countries prefer the use of first generation cephalosporins or a combination of penicillin and betalactamase-inhibitors $[18,20,23,24,25]$. Studies, however, have shown no difference between the use of broad spectrum and narrow spectrum prophylactic antibiotics in terms of lowering rate of SSI [12].

More gram-negative organisms were identified in isolates as compared to gram positive organisms which may be expected as a large proportion of patients underwent gastrointestinal surgery [26]. However, we found high rates of gram positive resistance, which may explain the high usage of vancomycin in our setting. These MDR organisms, particularly the gram positive organisms, should be closely monitored to evaluate the effectiveness of ASP initiatives in the future.

The SSI rate per patient observed in our study was similar to a previous local study [27]; but higher than the overall SSI rate reported by others $(1.2 \%-8.3 \%)$ $[19,25,28,29]$ except for India (20.1\%) [30]. The reason for this is uncertain but the impact of a high prevalence of inappropriate antibiotic use for prophylaxis, current infection control policies or a combination of these factors requires further consideration.

Inappropriate antibiotic use was not found to have significant impact on hospitalization days and allcause mortality in the current study though the high readmission rate due to relapse of infection and sepsis as a reported cause of death are of concern. We are unable to ascertain the direct role that inappropriate antibiotic use plays in these occurrences.

The aim of our Malaysian institution, and likely that of many others in newly industrialised countries, is to improve the appropriateness of antibiotic prescribing. As discussed, measures such as instituting local guidelines, improving documentation, standardisation of forms and education of prescribers may be sufficient to improve practices. These interventions can be performed in the absence of the resources that may be available in more developed nations.

One limitation of the study was that it was conducted at a single tertiary hospital and thus, the results may not be generalizable. We were also not able to sub-analyse the use of antibiotics according to surgical disciplines for prophylactic use due to the small number of prescriptions.
The strength of our study was that for the first time in Malaysia, we obtained insight on the use of antibiotics in a surgical setting. The resultant awareness has led to collaboration between the surgeons and the infectious disease consultants in developing guidelines for antibiotic use in surgery. Additionally, our institution has agreed to implement an ASP, to increase the appropriateness of antibiotic use in surgery.

\section{Conclusion}

Misuse of therapeutic antibiotics (42.0\%) and prophylactic antibiotics $(66.3 \%)$ was observed among surgical patients, further emphasizing an urgent need for antibiotic stewardship initiatives in this setting. A similar scenario is likely to be occurring in other developing nations, and rational use of antibiotics must be ensured to allow for optimization of healthcare outcomes for all patients.

\section{Acknowledgements}

We would like to thank the staff from the two surgical wards of University Malaya Medical Centre for their assistance during the study. Funding for the work was obtained from University of Malaya Research Grant (RG452-12HTM).

\section{Authors' contributions}

MK Lim, PSM Lai, CM Italiano, DCM Kong, NA Taib, A Kamarulzaman conceived the study and participated in the design; MK Lim, S Ponnampalavanar, CM Italiano, SF Syed Omar collected data; MK Lim, PSM Lai, S Ponnampalavanar, CM Italiano, SF Syed Omar performed the statistical analysis; MK Lim drafted the manuscript; all authors read and approved the final manuscript.

\section{References}

1. No authors listed (2014) Newly industrialized country. Available from: http://en.wikipedia.org/wiki/Newly_industrialized_country. Accessed on 20 December, 2014.

2. Dohmen PM (2008) Antibiotic resistance in common pathogens reinforces the need to minimise surgical site infections. J Hosp Infect 2: 15-20.

3. Classen DC, Evans RS, Pestotnik SL, Horn SD, Menlove RL, Burke JP (1992) The timing of prophylactic administration of antibiotics and the risk of surgical-wound infection. N Engl J Med 326: 281-286.

4. American Society of Health-System Pharmacists (1999) ASHP Therapeutic Guidelines on Antimicrobial Prophylaxis in Surgery. Am J Health Syst Pharm 56: 1839-18885.

5. Whitehouse JD, Friedman ND, Kirkland KB, Richardson WJ, Sexton DJ (2002) The impact of surgical-site infections following orthopedic surgery at a community hospital and a university hospital: adverse quality of life, excess length of 
stay, and extra cost. Infect Control Hosp Epidemiol 23: 183189.

6. Fukatsu K, Saito H, Matsuda T, Ikeda S, Furukawa S, Muto T (1997) Influences of type and duration of antimicrobial prophylaxis on an outbreak of methicillin-resistant Staphylococcus aureus and on the incidence of wound infection. Arch Surg 132: 1320-1325.

7. Laxminarayan R1, Duse A, Wattal C, Zaidi AK, Wertheim HF, Sumpradit N, Vlieghe E, Hara GL, Gould IM, Goossens H, Greko C, So AD, Bigdeli M, Tomson G, Woodhouse W, Ombaka E, Peralta AQ, Qamar FN, Mir F, Kariuki S, Bhutta ZA, Coates A, Bergstrom R, Wright GD, Brown ED, Cars O (2013) Antibiotic resistance - the need for global solutions. The Lancet Infectious Diseases 13: 1057-1098

8. Seni J, Najjuka CF, Kateete DP, Makobore P, Joloba ML, Kajumbula H, Kapesa A, Bwanga F (2013) Antimicrobial resistance in hospitalized surgical patients: a silently emerging public health concern in Uganda. BMC Res Notes 6: 1756-0500.

9. Lye DC, Earnest A, Ling ML, Lee TE, Yong HC, Fisher DA, Krishnan P, Hsu LY (2012) The impact of multidrug resistance in healthcare-associated and nosocomial Gramnegative bacteraemia on mortality and length of stay: cohort study. Clin Microbiol Infect 18: 502-508.

10. Dellit TH, Owens RC, McGowan JE Jr, Gerding DN, Weinstein RA, Burke JP, Huskins WC, Paterson DL, Fishman NO, Carpenter CF, Brennan PJ, Billeter M, Hooton TM; Infectious Diseases Society of America; Society for Healthcare Epidemiology of America (2007) Infectious Diseases Society of America and the Society for Healthcare Epidemiology of America guidelines for developing an institutional program to enhance antimicrobial stewardship. Clin Inf Dis : an official publication of the Infectious Diseases Society of America. 44: 159-77.

11. Ministry of Health Malaysia (2008) National antibiotic guidelines 2008 [cited 2014]; Available from: http://apps.who.int/medicinedocs/documents/s17800en/s1780 0en.pdf. Accessed on June 30, 2014.

12. Bratzler DW, Dellinger EP, Olsen KM, Perl TM, Auwaerter PG, Bolon MK, Fish DN, Napolitano LM, Sawyer RG, Slain D, Steinberg JP, Weinstein RA; American Society of HealthSystem Pharmacists; Infectious Disease Society of America; Surgical Infection Society; Society for Healthcare Epidemiology of America (2013) Clinical practice guidelines for antimicrobial prophylaxis in surgery. Am J Health Syst Pharm 70: 195-283.

13. (2011) Guidelines for ATC classification and DDD assignment 2012. WHO Collaborating Centre for Drug Statistics Methodology; Available from: http://www.whocc.no/filearchive/publications/2012_guideline s_with_front_pa.pdf Accessed Feb 27, 2015

14. Magiorakos AP, Srinivasan A, Carey RB, Carmeli Y, Falagas ME, Giske CG, Harbarth S, Hindler JF, Kahlmeter G, OlssonLiljequist B, Paterson DL, Rice LB, Stelling J, Struelens MJ, Vatopoulos A (2012) Multidrug-resistant, extensively drugresistant and pandrug-resistant bacteria: an international expert proposal for interim standard definitions for acquired resistance. Clin Microbiol Infect 18: 268-281.

15. Vessal G, Namazi S, Davarpanah MA, Foroughinia F (2011) Evaluation of prophylactic antibiotic administration at the surgical ward of a major referral hospital, Islamic Republic of Iran. East Mediterr Health J 17: 663-668.
16. Al-Momany NH, Al-Bakri AG, Makahleh ZM, Wazaify MM (2009) Adherence to international antimicrobial prophylaxis guidelines in cardiac surgery: a Jordanian study demonstrates need for quality improvement. J Manag Care Pharm 15: 262271.

17. Parulekar L, Soman R, Singhal T, Rodrigues C, Dastur FD, et al. (2009) How good is compliance with surgical antibiotic prophylaxis guidelines in a tertiary care private hospital in India? A prospective study. Indian J Surg 71: 15-18.

18. Miliani K, L'Heriteau F, Astagneau P (2009) Non-compliance with recommendations for the practice of antibiotic prophylaxis and risk of surgical site infection: results of a multilevel analysis from the INCISO Surveillance Network. J Antimicrob Chemother 64: 1307-1315.

19. Choi WS, Song JY, Hwang JH, Kim NS, Cheong HJ (2007) Appropriateness of antibiotic prophylaxis for major surgery in Korea. Infect Control Hosp Epidemiol 28: 997-1002.

20. Napolitano F, Izzo MT, Di Giuseppe G, Angelillo IF (2013) Evaluation of the appropriate perioperative antibiotic prophylaxis in Italy. PLoS One 8.

21. Mahdaviazad H, Masoompour SM, Askarian M (2011) Iranian surgeons' compliance with the American Society of Health-System Pharmacists guidelines: antibiotic prophylaxis in private versus teaching hospitals in Shiraz, Iran. J Infect Public Health 4: 253-259.

22. Hosoglu S, Aslan S, Akalin S, Bosnak V (2009) Audit of quality of perioperative antimicrobial prophylaxis. Pharm World Sci 31: 14-17.

23. Durando P, Bassetti M, Orengo G, Crimi P, Battistini A, Bellina D, Talamini A, Tiberio G, Alicino C, Iudici R, Sticchi C, Ansaldi F, Rossi A, Rosso R, Viscoli C, Icardi G; Surgical Audit Team of the San Martino University Hospital of Genoa (2012) Adherence to international and national recommendations for the prevention of surgical site infections in Italy: results from an observational prospective study in elective surgery. Am J Infect Control 40: 969-972

24. Hohmann C, Eickhoff C, Radziwill R, Schulz M (2012) Adherence to guidelines for antibiotic prophylaxis in surgery patients in German hospitals: a multicentre evaluation involving pharmacy interns. Infection 40: 131-137.

25. Young B, Ng TM, Teng C, Ang B, Tai HY, et al. (2011) Nonconcordance with surgical site infection prevention guidelines and rates of surgical site infections for general surgical, neurological, and orthopedic procedures. Antimicrob Agents Chemother 55: 4659-4663.

26. Singh AV, Mishra B, Thakur A (2009) Multidrug-resistant Gram-negative bacteria in postoperative infections. J Indian Med Assoc 107: 148-150, 163.

27. Oh AL, Goh LM, Nik Azim NA, Tee CS, Shehab Phung CW (2014) Antibiotic usage in surgical prophylaxis: a prospective surveillance of surgical wards at a tertiary hospital in Malaysia. J Infect Dev Ctries 8: 193-201.

28. Diaz-Agero Perez C, Robustillo Rodela A, Pita Lopez MJ, Lopez Fresnena N, Monge Jodra V (2014) Surgical wound infection rates in Spain: Data summary, January 1997 through June 2012. Am J Infect Control 18: 01458-01452.

29. Kasatpibal N, Jamulitrat S, Chongsuvivatwong V (2005) Standardized incidence rates of surgical site infection: a multicenter study in Thailand. Am J Infect Control 33: 587594.

30. Mahesh CB SS, Suresh BS, Chidanand SP, Vishwanath Y (2010) A prospective study of surgical site infections in a teaching hospital. J Clin Diagn Res 4: 3114-3119. 


\section{Corresponding author}

Dr Pauline Siew Mei LAI

Department of Primary Care Medicine

Faculty of Medicine

University of Malaya

50603 Kuala Lumpur

Malaysia

Phone: +60379493920

Email: plai@ummc.edu.my

Conflict of interests: DCMK has sat on advisory boards for Pfizer and Merck, Sharp \& Dohme, and receives financial support (all not related to the current work) from Pfizer, Roche, Novartis, Merck, Sharp \& Dohme, and Gilead Sciences.. 